\title{
Shakespeare et la gravure morale de l'Europe du Nord
}

Josée Nuyts-Giornal

\section{(2) OpenEdition \\ Journals}

\section{Édition électronique}

URL : http://journals.openedition.org/shakespeare/758

DOI : 10.4000/shakespeare.758

ISSN : 2271-6424

Éditeur

Société Française Shakespeare

\section{Édition imprimée}

Date de publication : 1 novembre 2005

Pagination : 203-220

ISBN : 2-9521475-1-5

\section{Référence électronique}

Josée Nuyts-Giornal, «Shakespeare et la gravure morale de l'Europe du Nord », Actes des congrès de la Société française Shakespeare [En ligne], 22 | 2005, mis en ligne le 01 janvier 2007, consulté le 20 avril 2019. URL : http://journals.openedition.org/shakespeare/758 ; DOI : 10.4000/shakespeare.758 


\section{Shakespeare et l'Europe de la Renaissance}

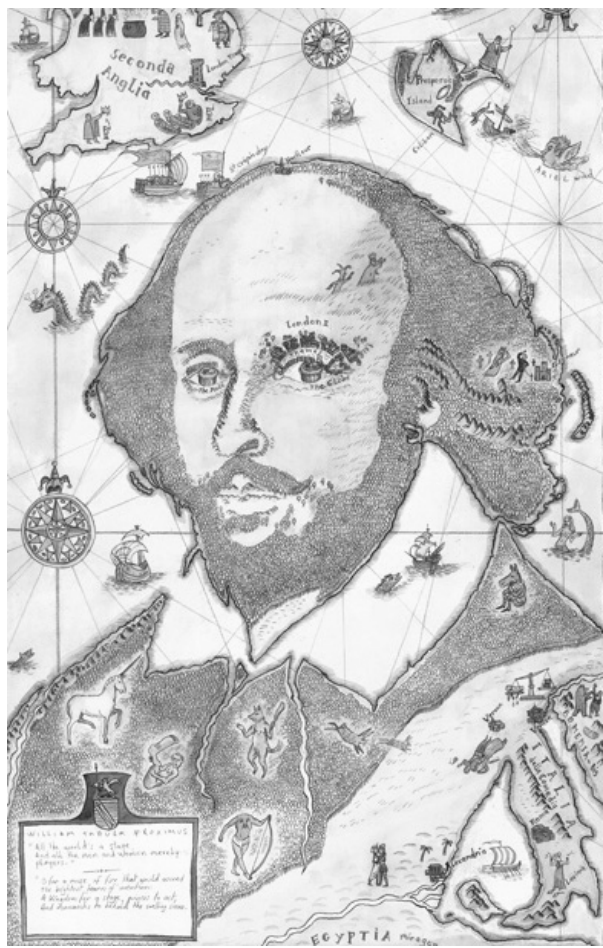

a ctes du Congrès

organisé par la

SOCIÉTÉ FRANÇAISE SHAKESPEARE

les 11,12 et 13 mars 2004

textes réunis par

Pierre KAPITANIAK

sous la direction de

Yves PEYRÉ 


\title{
COMITÉ SCIENTIFIQUE :
}

\author{
Margaret Jones-Davis \\ Jean-Marie Maguin \\ Yves Peyré \\ Pierre Kapitaniak
}

COUVERTURE :

Edouard Lekston, William Tabula Proximus, 2004

conception graphique et logo

Pierre Kapitaniak

CC 2004 Société Française Shakespeare

Institut du Monde Anglophone

Université de Paris III - Sorbonne Nouvelle

http:// univ-montp3.fr/SFS/

5 rue de l’École de Médecine

75006 Paris

ISBN 2-952 1475-1-5

Tous droits de traduction, de reproduction et d'adaptation réservés pour tous les pays 


\title{
SHAKESPEARE ET LA GRAVURE MORALE DE L'EUROPE DU NORD
}

\author{
Josée NUYTS-GIORNAL
}

\begin{abstract}
À la Renaissance, l'humanisme nordique se trouvait au cœur d'une imagerie collective importante employée par le théâtre et l'art visuel. La gravure néerlandaise, véritable lexique des schémas majeurs de la pensée humaniste, semble à même de révéler les faces inattendues de certaines tournures verbales courantes à l'époque. L'univers pictural de Pieter Bruegel l'ancien, Cornélis Anthonisz, Maarten van Heemskerck, et David Vinckboons, parmi d'autres, porte la trace de ses relations avec le discours humaniste sur l'art visuel et les réjouissances populaires. La préférence prodigieuse pour les estampes flamandes et néerlandaises au XV| ${ }^{e}$ siècle, observée par Anthony Wells-Cole dans Art and Decoration in Elizabethan and Jacobean England pourrait s'expliquer par le ton moralisateur caractéristique de la gravure de genre nordique. Les références récurrentes à l'art pictural chez Shakespeare et ses contemporains témoignent de l'étendue de cette imagerie typique. Aussi, l'analyse de certains thèmes et motifs propres à l'art pictural dans le texte shakespearien permettra d'entrevoir la morale à travers des perspectives parfois obliques.
\end{abstract}

Shakespeare, Early Modern Moral Topoi, and Dutch Prints Lately a renewed interest in Northern Humanism with its particularities and its relation to sixteenth-century literature and theatre has been the subject of some interesting studies. Yet it appears that Dutch moral engraving, a virtual lexicon of some major humanist thought schemes, still has some intriguing patterns to disclose. The position of Dutch prints as a vector and recipient of Northern Humanism at large can be seen as consonant with the model of Fernand Braudel adapted by Peter Burke to explain areas of cultural influence and homogeneity. The pictorial worlds of Pieter Bruegel, Cornélis Anthonisz, Maarten van Heemskerck, Hendrick Goltzius, David Vinckboons among others bear witness to their links with humanist discourse concerning visual art, theatre and popular amusement. When taking a closer look at particular visual themes and motifs it happens that prints help to decipher some of the playwright's verbal imagery. Ambiguous passages in several of Shakespeare's plays and in those of his fellow playwrights appear to be allusions to thematic issues also dealt with in moral engraving. This paper will give a succinct outline of Dutch engraving as a moral phenomenon before giving a short analysis of some major motifs partly based on my previous research on the relation between moral prints and Elizabethan drama.

$\mathrm{A}$ la Renaissance, l'humanisme des pays de l'Europe du Nord avait donné naissance à des images que l'on retrouve en Angleterre dans le théâtre et les arts visuels. L'étude des liens entre l'humanisme, le théâtre et la littérature du XVI ${ }^{\mathrm{e}}$ siècle a donné lieu à de nombreuses publications récentes qui jettent un éclairage nouveau sur ces questions ${ }^{1}$. La gravure néerlandaise, véritable lexique des schémas majeurs de la pensée humaniste est, en effet, d'un secours précieux dans la mesure où elle est à même de révéler les faces inattendues de certaines tournures verbales courantes à l'époque. Le rôle de vecteur et de réceptacle de la pensée humaniste au sens large

\footnotetext{
${ }^{1}$ Kent Cartwright, Theatre and Humanism, English Drama in the Sixteenth Century, Cambridge, Cambridge University Press, 1999 ; Robert Bennet, Rom ance and Reformation, The Erasmian Spirit of Shakespeare's Measure for Measure, Newark / Londres, University of Delaware Press, Associated University Presses, 2000.
} 
qu'occupe l'estampe néerlandaise est conforme au modèle de Fernand Braudel adopté par Peter Burke à propos d'aires d'influence et d'homogénéité culturelle ${ }^{2}$. L'univers pictural de Bruegel l'Ancien, de Cornélis Anthonisz, de Maarten van Heemskerck, et de David Vinckboons, parmi bien d'autres, porte en effet la marque du discours humaniste ambiant sur l'art visuel et les réjouissances populaires.

$\mathrm{Au}$ siècle dernier, l'interdépendance entre les moyens de communication verbaux et visuels au début de l'époque moderne fut l'objet d'un nombre important d'études. Dans le prolongement de l'œuvre d'Erwin Panofsky, Quentin Skinner part d'un cycle de fresques d'Ambrogio Lorenzetti afin de saisir les «idées en contexte » dans son essai d'histoire de la philosophie politique. L'essai présenta une double rupture méthodologique. Tout d'abord, il remit en cause la prééminence du texte dans l'histoire des idées, puis l'auteur y inversa la lecture traditionnelle de l'iconographie politique. En effet, ici, l'image conduit aux textes, car elle semble parfois mieux à même de rendre compte d'une tradition philosophique ou idéologique où se mêlèrent des apports théoriques en apparence dissemblables et disparates, en partie occultés aujourd'hui ${ }^{3}$. L'artiste apparaît ainsi en philosophe de son temps. Aussi, dans leur étude du texte shakespearien, Roland Mushat Frye, Rosemary Wright et John H. Astington ont-ils eu recours à certaines estampes choisies ${ }^{4}$. Alastair Fowler, dans un ouvrage plus récent, s'appuie sur l'art visuel afin de redéfinir les conventions d'un réalisme narratif propre à la Renaissance. Notre étude ${ }^{5}$, évoquant la gravure septentrionale en tant que phénomène, puis éclairant quelques thèmes majeurs, se situe dans cette lignée tout en renvoyant à ma thèse

\footnotetext{
${ }^{2}$ Peter Burke, Antwerp, A Metropolis in Comparative Perspective, Anvers, Martial \& Snoeck, 1993.

${ }^{3}$ Quentin Skinner, L'artiste en philosophe politique, Ambrogio Lorenzetti et le Bon Gouvernement, trad. Rosine Christin, Paris, Raisons d'Agir, Cours et Travaux, 2003, p. 11, $1^{\mathrm{re}}$ éd. Ambrogio Lorenzetti : The Artist as a Political Philosopher, New York, Barnes and Noble, 1986.

${ }^{4}$ Roland Mushat Frye, «Ladies, Gentlemen, and Skulls: Hamlet and the Iconographic Traditions », Shakespeare Quarterly, vol. 30, ${ }^{\circ}{ }^{1}$, hiver 1979, p. 15-28 ; Rosemary Wright, «Prospero's Lime Tree and the Pursuit of Vanitas », Shakespeare Survey, 37, 1984; John H. Astington, "Three Shakespearean Prints", Shakespeare Quarterly, vol. 47, n², été 1996, p. 178-189.

${ }^{5}$ Elle n'aborde pas la recherche d'images sources telle qu'elle a été entreprise par Anthony Wells-Cole, entre autres, et telle qu'elle a été relevée par Tessa Watt à propos des estampes sources d'inspiration pour le « painted cloth », un autre sujet passionnant.
} 
de doctorat sur la gravure néerlandaise et le drame élisabéthain, intitulée «Le miroir de la folie».

L'extraordinaire engouement $\mathrm{du} \mathrm{XVI}{ }^{\mathrm{e}}$ siècle pour les estampes flamandes et néerlandaises, que relève par Anthony Wells-Cole dans son ouvrage, Art and Decoration in Elizabethan and Jacobean England, pourrait s'expliquer par le ton moralisateur caractéristique de la gravure de genre nordique :

it may be that the moralizing overtones of many of them struck a sympathetic cord in English society. For after the abolition of the confessional at the Reformation, there was what has been described as a widespread conviction that the unaided protestant conscience was an inadequate sanction for morality ${ }^{6}$.

L'intensité morale des textes de John Skelton et de Sir Thomas Wyatt avait déjà été rapprochée de l'influence flamande par David Evett. George Kernodle, quant à lui, mentionne également une tendance moralisatrice similaire qui sévissait des deux côtés de la Manche.

Les références récurrentes à l'art pictural chez Shakespeare et ses contemporains témoignent de l'étendue de cette imagerie morale ainsi que de sa nature conventionnelle ${ }^{7}$, particulièrement bien exprimées dans les passages suivants :

Who fears a sentence or an old man's saw

Shall by a painted cloth be kept in awe.

The Rape of Lucrece, v. 244-245

ORLANDO. I answer you right painted cloth from whence you have studied your questions.

As You Like It, III.i.268

L'étude de l'art pictural nordique semble dès lors particulièrement fondée dans l'analyse littéraire et vice-versa. Beaucoup d'articles et ouvrages concernent la tradition des emblèmes. Cependant les images morales auxquelles font référence Shakespeare ou John Donne semblent appartenir à un corpus moins tangible: tableaux, estampes sur feuille volante, tissus peints ou séries de «brave pictures ${ }^{8}$ », ornant l'intérieur des maisons comme dans certains tableaux de genre. Les

\footnotetext{
${ }^{6}$ Anthony Wells-Cole, Art and Decoration in Elizabethan and Jacobean England, New Haven, Yale University Press, 1998, p. 300.

7 «A thousand moral paintings I can show (Tim on of Athens, I.i.91).

8 Ben Jonson, Bartholomew Fair, (III.v), Complete Plays, Londres, Everyman's Library, 1969.
} 
images reproduites par Anthony Wells-Cole pourraient, en partie, constituer un tel corpus. Dans Cheap Print and Popular Piety, 15501640, Tessa Watt tente d'imaginer une taverne métaphorique selon les théories mnémotechniques décrites par Francis Yates ${ }^{9}$. L'auteur admet cependant aussitôt que la dominante pieuse du modèle obtenu est sans doute exagérée. Car n'y figurent plus les images moins respectables et, de ce fait, moins respectées, qui n'ont donc pas survécu aux aléas du temps. Le témoignage de l'historien d'art Ger Luijten va également dans ce sens, à l'aide de l'exemple révélateur de l'inventaire d'un chargement de gravures en partance vers un comptoir hollandais en 1600. Aux côtés d'une majorité de scènes profanes, estampes de Jaques de Gheyn et un fils prodigue de Karel van Mander, on trouve en effet «a remarkable number of fools, drunkards and peasants, together with women depicted in an unflattering light ${ }^{10} »$. S'agit-il ici des «drolleries» que Falstaff apprécie sur un mur de taverne, terme également employé pour désigner les estampes satiriques de Bruegel l'ancien? Dans Satyre IIII, John Donne semble effectivement faire référence à ce type d'images caractéristiques de l'estampe de genre des pays du Nord :

To teach by painting drunkards, doth not last

Now Aretine's pictures have made few chaste;

No more can Princes courts, though there be few, Better pictures of vice teach me virtue ${ }^{11}$.

Dans Summer's Last Will and Testament, Thomas Nashe fait également une allusion ironique à ce type de contre-exemple ou negative self-definition :

Drunkennesse of his good behaviour

Hath testimonial from where he was borne:

That pleasant worke de arte bibendi,

Adrunken Dutchman spued out a few years since... $\quad(1404-07)^{12}$

\footnotetext{
${ }^{9}$ Tessa Watt, Cheap Print and Popular Piety, 1550-1640, Cambridge, Cambridge University Press, 1991, p. 332. Francis A. Yates, L'art de la mémoire, ler éd. 1966, trad. Daniel Arasse, Paris, Gallimard, 1975.

${ }^{10}$ Dawn of the Golden Age, Northern Netherlandish Art, 1580-1620, Catalogue Rijksmuseum Amsterdam, Waanders, Zwolle, 1994, p. 183.

${ }^{11}$ John Donne, Satyre IIII, 69-72, The Works of John Donne, coll. The Wordsworth Poetry Library, Londres, Wordsworth Editions, 1994.

12 The Works of Thomas Nashe, éd. Ronald B. McKerrow, vol. III, Oxford, Basil Blackwell, 1966. Référence possible à l'ouvrage Drunkardes mentionné dans English Woodcuts 1480-
} 
De la même manière, la récurrence de certains thèmes et motifs propres à l'art pictural dans le texte shakespearien permet de faire entrevoir une morale par le biais de perspectives parfois obliques.

Même si une certaine réticence subsiste du fait de l'excès des commentaires sur le détail symbolique, critiques et historiens d'art concèdent aujourd'hui que la gravure et la peinture de genre, objet de lassitude chez Donne, contiennent des admonitions morales. Le concept du miroir de la folie auquel se réfère le poète est essentiel dans l'art graphique du nord à partir du $X^{e}$ siècle, et offre de nombreux points de convergence avec le théâtre séculier de la même époque. La tradition iconographique médiévale était en partie issue des illustrations des dix commandements. Ces images fondées sur la négation, «tu ne tueras point, tu ne convoiteras point », représentaient les ignominies à éviter, tout en suscitant par là même un goût certain pour des scènes de transgression et d'excès. Ainsi, si les représentations du fils prodigue montraient tout d'abord les différents épisodes de la fable avec une attention égale, au $\mathrm{XVI}^{\mathrm{e}}$ siècle, les scènes de taverne présentant le protagoniste en compagnie d'êtres dissolus prirent progressivement le pas sur les scènes de punition et de repentance, et cela dans l'art pictural comme sur la scène théâtrale. À partir de 1530, la vie dissolue du Prodigue était établie comme un sujet indépendant, l'image de repentance, parfois reléguée à l'arrière-plan, restant cependant sous-entendue ${ }^{13}$. À la même époque, le motif iconographique des compagnies joyeuses ou «merry companies», sans lien direct avec la parabole biblique, voit le jour. Le Banquet nocturne d'après Joos van Winghe, rappelant les paroles de John Donne dans Satyre IIII en est un exemple (ill. I).

Ces images satiriques de la folie humaine se trouvent dans la lignée des pécheurs joyeux, sur une barque ou sur une meule de foin dépeints par Jérôme Bosch. Ces images allégoriques sont fondées sur un écart ironique entre l'image et sa lecture. Par cette double perspective inhérente à ces représentations singulières, l'image de la folie se condamne elle-même, l'expiation dans l'autre monde étant

1535, Oxford, Edward Hodnett, 1973, p. 65-66. Le livre hollandais fut traduit en anglais en 1523 , et comportait 17 gravures sur bois illustrant des scènes de l'Ancien Testament afin d'illustrer une morale de tempérance.

13 Dans A Mad World, My Masters, Thomas Middleton semble donner un commentaire burlesque sur cette tendance: «The curtains were wrought in Venice with the story of the Prodigal child in silk and gold; only the swine were left out for spoiling the curtains » (III.ii.5-8). 
inévitable. Le jugement moral est parfois figuré par un bouffon, observateur et commentateur goguenard de la scène représentée, comme dans le Fils Prodigue d'après Karel van Mander (ill. II). D'une manière ironique, les apartés du bouffon rappellent le beschouwer ou spreker habillant de son commentaire les scènes allégoriques des processions de rue. Des exemples de ce phénomène de lecture de l'image de la folie sont récurrents chez Shakespeare. Ainsi, dans All's Well That Ends Well, Bertram s'exclame, confronté au comportement affligeant de l'impénitent Parolles : «I know his brains are forfeit to the next tile that falls» (IV.iii.177) ${ }^{14}$. Ce passage est par ailleurs situé par le dramaturge dans un contexte d'estampe de genre par son intitulé : « dialogue between the Fool and the Soldier» (IV.iii.101). Falstaff, stéréotype du vieux et sot pécheur également présent dans l'estampe de genre, est condamné de façon similaire dans l'épilogue de 2 Henry IV: «Where, for anything I know, Falstaff shall die of a sweat, unless already a be killed with your hard opinions. »

$\mathrm{Au} \mathrm{XVI}^{\mathrm{e}}$ siècle un certain conservatisme règne dans la représentation iconographique des motifs et concepts moraux. La réimpression de planches d'artistes du $\mathrm{XV}^{\mathrm{e}}$ et $\mathrm{XVI}^{\mathrm{e}}$ siècles jusqu'au XVII siècle a indéniablement contribué à cet effet, mais il y avait également une importante continuité thématique. Le propos moral exprimé dans des séries de gravures telle que Sinful Mankind before and After the Flood, de Jan Sadeler I d'après Dirk Barendsz, semble faire écho aux commentaires de certains protagonistes shakespeariens, comme les craintes exprimées par le roi Henri IV à propos du comportement à venir de son fils : «commit the oldest sins the newest kind of ways?» (IV.iv.125-28) ${ }^{15}$. Le topos du miroir de la folie avec ses ramifications thématiques, comme les gravures de proverbes de Bruegel l'Ancien, appelé aussi monde à l'envers, participe de ce que Paul Zumthor appelle «une emblématique sociale ${ }^{16}$ ». Discours éthique fondé sur le lieu commun, la sagesse proverbiale et le folklore populaire s'y mêlent

\footnotetext{
14 Dans A Choice of Emblems (1586) de Geffrey Whitney, le comportement dissolu d'une jeune femme jouant aux dés, est puni de façon similaire : « But loe a tyle upon her head did fall » (A Choice of Em blemes, Aldershot, Scolar Press, 1989, intr. John Manning).

15 «PRINCE HALL. I am now of all humours that have showed themselves humours since the old days of goodman Adam to the pupil-age of this present twelve o'clock at midnight » (II.iv.94-6) in The Oxford Shakespeare, The Complete Works, éd. Stanley Wells, Gary Taylor, Oxford, Clarendon Press, 1994.

16 Paul Zumthor, Le Masque et la Lumière, Paris, Seuil, 1978, p. 153.
} 


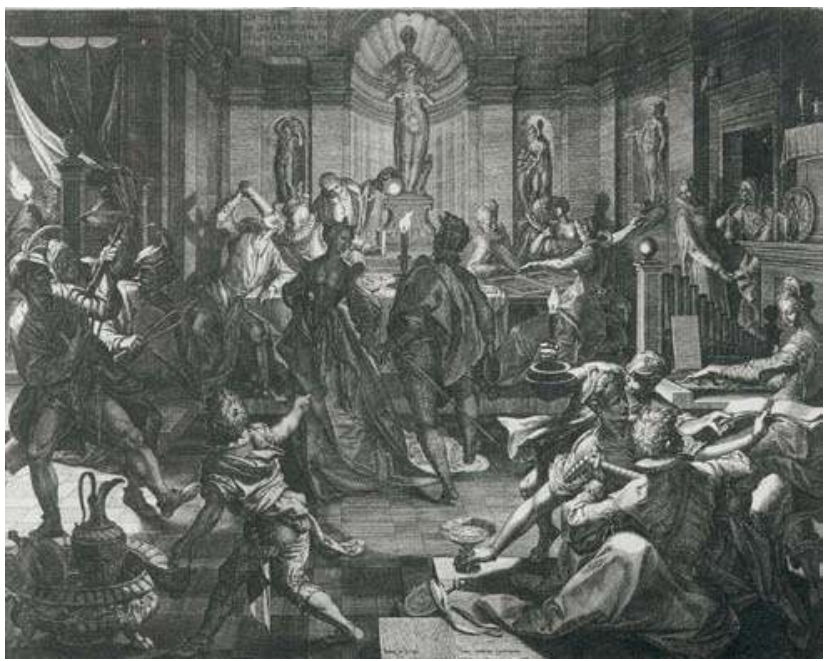

I Banquet Nocturne avec masquarade, 1588, Johannes Sadeler d'après Joos van Winghe (Rijksprentenkabinet, Cabinet d'estampes, Amsterdam).

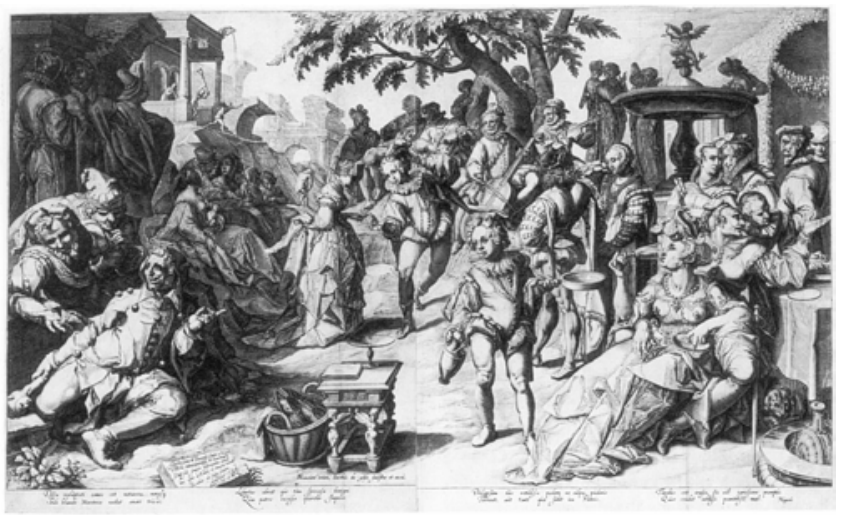

II Le Fils prodigue avec bouffon, 1596, Karel van Mander (Rijksprentenkabinet, Cabinet d'estampes, Amsterdam). 


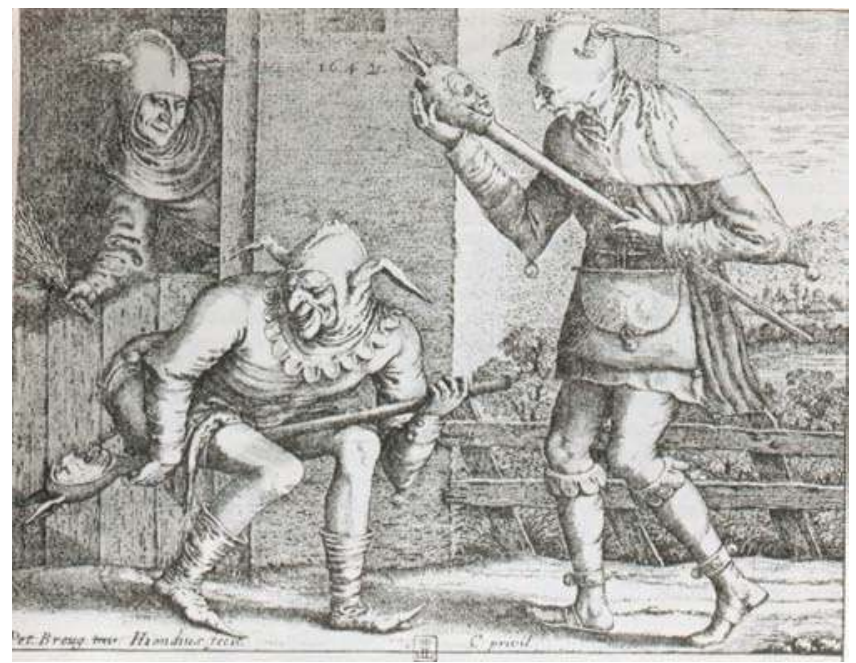

III Trois bouffons, Hendrick Hondius d'après Bruegel l'Ancien (Musée Boijmans van Beuningen, Rotterdam).

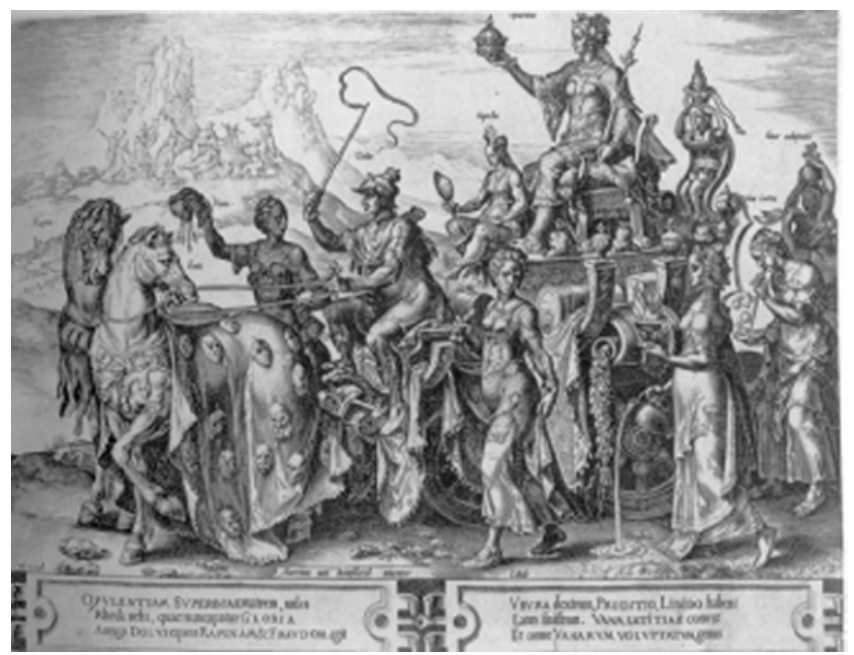

IV De la richesse naît l'orgueil dans la série Le Cycle des agissements humains, d'après Maarten van Heemskerck (Rijksprentenkabinet, Cabinet d'estampes, Amsterdam). 


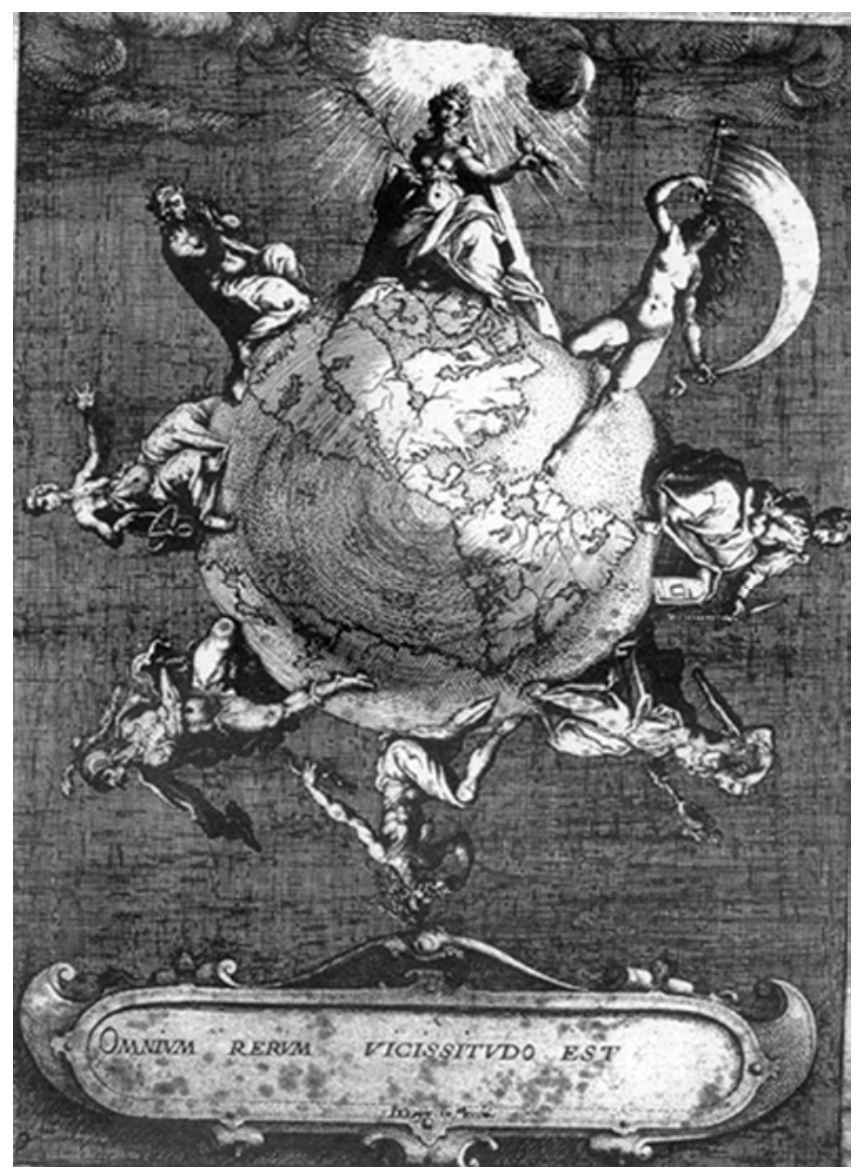

V Omnium rerum vicissitudo est, 1597 ,

Zacharias Dolendo d'après Jacques de Geyhn

(Cabinet d'estampes de l'Université de Leiden). 


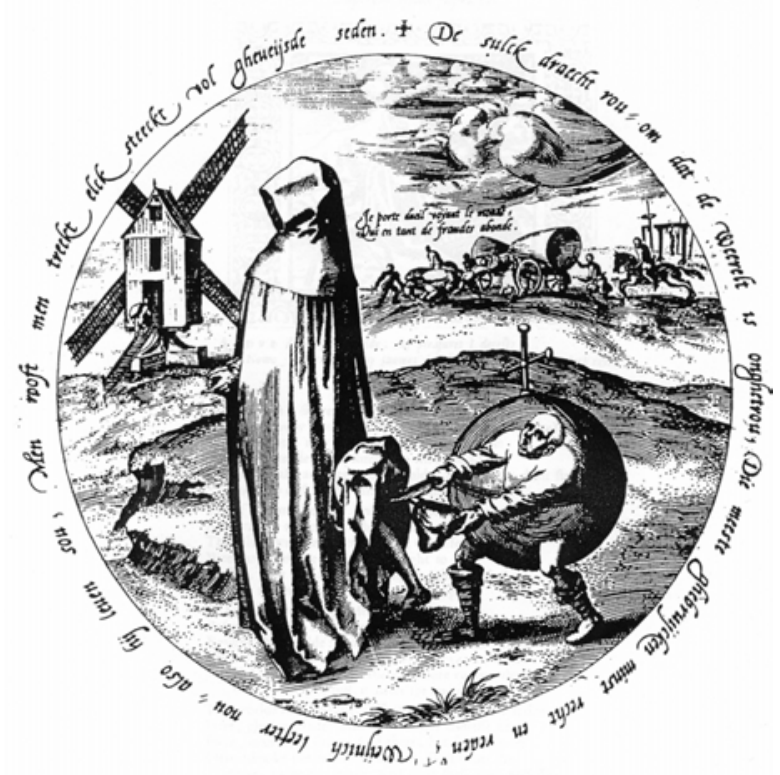

VI Le misanthrope, proverbe en tondo, d'après Bruegel l'Ancien (Rijksprentenkabinet, Cabinet d'estampes, Amsterdam).

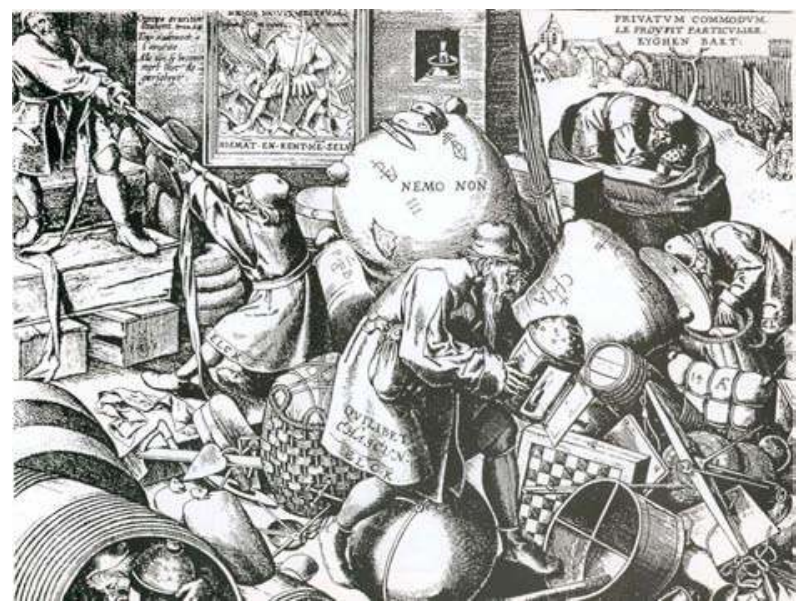

VII Elck ou Chascun, H. Cock d'après Bruegel l'Ancien (Musée Boijmans van Beuningen, Rotterdam). 
aux exempla moraux classiques un peu à la manière des marginalia dans les livres d'emblèmes.

Dans sa traduction de l'ouvrage de Sebastian Brant, The Shyp of Folys of the World (1509), Alexander Barclay expose l'idée commune de miroir moral «for man to behold his life and misgoverned manners », dont les réminiscences subsistent dans la présentation du personnage Shakespearien d'Antony: «The triple pillar of the world transformed into a strumpet's fool. Behold and see»(I.i.12). The Anatomy of Melancholy est fondée sur le même principe ayant envahi le discours humaniste septentrional à la Renaissance. En effet, Robert Burton invite le lecteur à se reconnaître dans le portrait d'un aliéné ${ }^{17}$. Cependant, lorsque John Milton loua Edmund Spenser pour la valeur didactique de sa poésie visuelle, surpassant l'enseignement de Jean Duns Scot ou de Thomas d'Aquin, en ayant peint les dangers de la luxure dans les jardins d'Acrasia pour que Guyon «might see and know, and yet abstain ${ }^{18} »$, l'argument avait déjà été subverti. Ainsi, dans A Trick to Catch the Old One, Thomas Middleton adapta l'idée afin qu'elle convienne à la Courtisane : «She that knows sin knows best how to hate sin.» Les scènes de taverne dans les deux parties d'Henry IV devraient sans doute être lues sous l'angle d'une éducation humaniste du Prince ${ }^{19}$, bien que quelques libertés semblent avoir été prises avec le concept de départ. Le dramaturge paraît faire écho à la polémique contemporaine concernant le théâtre et les arts visuels, qui portait sur la qualité édifiante des images d'excès ou, au contraire, la nature contagieuse de leur vivacité.

Les voies de la sagesse, tracées par les humanistes du Nord, semblent tout d'abord emprunter le chemin médiéval vers la rédemption. Mais l'équation traditionnelle entre péché et folie se trouva singulièrement amplifiée ${ }^{20}$. La fascination exercée par l'idée de

\footnotetext{
${ }^{17}$ Robert Burton, «Argument of the Frontispice», The Anatomy of Melancholy, 1e éd. 1621, Londres, Holbrook J ackson, 1977.

18 John Milton, Aeropagitica, 100-107, in Selected Shorter Poems and Prose, Londres, Routledge, 1988, (1 1 éd. 1644).

19 Josée Nuyts-Giornal, «Henri v ou l'entre-deux songes, portrait d'un prince humaniste », Théâtres du Monde, $\mathrm{n}^{\circ}$ 12, Avignon, Université d'Avignon, 2002, p. 65-84.

${ }^{20}$ La conception médiévale du Mal se mue en la conception humaniste de la déraison de l'homme, dont le libre arbitre n'est pas exclu. Ainsi le concept rentre dans le domaine de la connaissance et devient le postulat à partir duquel l'homme va penser sa vérité. Voir Michel Foucault, Histoire de la folie à l'âge classique, Paris, Gallimard, 1972, p. 24-25 ; JeanClaude Margolin, Érasme, Euvres choisies, Paris, Laffont, 1992, p. cxvii ; Shakespeare,
} 
la folie dans les cercles d'Érasme et de Thomas More donna lieu à une dialectique complexe, proche de celle des mock encomia exemplifiée par L'Éloge de la folie. Ce discours est fondé sur une vision ironique de l'humanité au cœur duquel se trouva la maxime de Sénèque, «When I wish to see a fool, I reflect upon myself, and there I have him », illustrée par Hans Holbein, dans la marge de l'Éloge, et citée par Robert Burton ${ }^{21}$. Le thème, constitutif d'une grande partie des gravures morales du Nord, se retrouve dans les comédies et tragédies de Shakespeare. Sans relâche, les protagonistes shakespeariens se voient confrontés à cette vérité première qu'est leur folie et celle de tous les hommes d'une manière ironique ou tragique. Dans Love's Labour's Lost, le dramaturge reprend l'image drolatique assez courante dans les arts visuels, présentant plusieurs fous narquois dévisageant le spectateur, obligé à se reconnaître comme étant le fou manquant dans le tableau. «That you three fools lack'd me fool to make up the mess » $(\text { IV.iii.202) })^{22}$. Le tempérament mélancolique mais facétieux de la Renaissance du Nord semble avoir uni l'adage de Socrate sur la nécessaire connaissance de soi, jusque-là correctement interprétée comme ayant trait aux capacités personnelles et position, et la maxime de Sénèque ${ }^{23}$. Dès lors, la seule connaissance significative accessible à l'homme est : «Know thyself to be a fool ${ }^{24}$ », ellipse pénétrante mais tragique dont le texte dramatique porte la trace. Elle constitue la plaisanterie dont Jaques est le dupe dans As You Like It, invitant à l'épreuve à laquelle sera soumis Hamlet, puis elle paraît au travers de la lamentation finale de Macbeth.

Comment le Mal vient aux Hommes, Actes du Congrès 1997, éd. Patricia Dorval, dir. JeanMarie Maguin, Paris, Société Française Shakespeare, 1997.

${ }^{21}$ Robert Burton, «Democritus to the Reader », The Anatomy of Melancholy, Londres, Holbrook Jackson, 1977, p. 77.

22 Cf. aussi Twelfth Night, (II.iii.10-16).

23 Ainsi, Robert Ascham relève «That wise proverbe of Apollo, Knowe Thyselve : that is to saye learne to knowe, what thou arte able fitte and apt unto, and follow that " (Toxophilus, 1545, p. 155). Voir Morris Palmer Tilley, A Dictionary of Proverbs in England in the $16^{\text {th }}$ and $17^{\text {th }}$ centuries, Ann Arbor, University of Michigan Press, 1966.

24 «The wise man knows himself to be a fool» (As You Like It, v.i.30). Dans Coriolanus, le dramaturge décrit la figure de façon détaillée. En effet, Menenius souhaite que les magistrats romains se soumettent à un exercice poussé d'introspection : «O that you could turn your eyes to the napes of your necks, and make but an interior survey of your good selves! Oh that you could! then you should discover a brace of unmeriting proud, violent, testy magistrates-alias fools- [...] You know neither me, yourselves or anything » (II.i.3675). 
L'antagonisme habituel entre vices et vertus, représenté dans les psychomachia de Prudence se trouva supplanté par la moriomachie, sujet récurrent dans l'art graphique. Ainsi, la Stultorum chorea de Frans Hogenberg, ou la Fête des fous d'après Bruegel l'Ancien. L'estampe attribuée à Bruegel montre une multitude de fous se moquant les uns des autres et rappelle la devise des sociétés de fous ou fraternités de la barque bleue du continent de la fin du XVe siècle : Stultorum numerus est infinitus. Ce thème visuel est repris jusqu'au $\mathrm{XVII}^{\mathrm{e}}$ siècle, ainsi dans la gravure de Hendrick Hondius datant de 1642 (ill. III). En Angleterre, Cock Lorrel's Bote, XX Orders of Fooles (156970), puis A New Ballad against Unthrifts, semblent fondés sur le même motif. All Fools (1599) de George Chapman est composé sur un ton satirique similaire, et propose une galerie de fous que le public est convié à rejoindre à la fin, à la manière des estampes facétieuses mentionnées. Évocatrice des satiristes classiques ${ }^{25}$, fréquemment invoqués afin de justifier ces portraits discourtois, la satire humaniste dessine les incartades de l'homme dans le dessein de tester la conscience morale, ou à défaut l'esprit / wit du public. À cet égard, le genre honorait Horace et la vertu réconciliée avec le plaisir, commenté de façon exhaustive à la Renaissance. Dans Theatre and Humanism, Kent Cartwright cite un critique contemporain proclamant, à l'instar d'Érasme, que les spectateurs ne devraient pas oublier leur jugement éthique lorsqu'ils se rendent au théâtre ${ }^{26}$. Le public élisabéthain était censé reconnaître les allusions et références aux motifs et concepts moraux communs à l'art pictural et à la littérature afin de deviner la perspective morale correcte. Ce principe humaniste semble clairement démontré dans le prologue à Summer's Last Will and Testament: «Moralizers, you that wrest a never-meant meaning out of everything, applying all things to present time, keep your attention for the common stage, for here are no quips in characters for you to read ${ }^{27}$. » La pièce de Thomas Nashe, fondée sur le lieu commun moral, fournit un inventaire ironique mais utile des motifs les plus récurrents, même si cela est tout naturellement nié par le bouffon.

\footnotetext{
25 Théophraste, Caractères ou Juvénal.

26 Kent Cartwright, op. cit., p. 195.

27 Thomas Nashe, Prologue, Summer's Last Will and Testament, in The Works of Thomas Nashe, vol. 3, éd. Ronald B. Mckerrow, Oxford, Basil Blackwell, 1966.
} 
De façon similaire, le texte de Shakespeare fait de multiples références à l'imagerie morale. Cependant, à travers ses emprunts burlesques ou subvertis, le dramaturge se moque parfois de leur nature conventionnelle et éclaire la limite de leurs réponses édifiantes. Afin de saisir ces états d'âme, souvent fondés sur l'écart par rapport à l'attendu, une connaissance des motifs initiaux semble requise. L'art de la perception demandée est proche du discernement contextuel nécessaire à la lecture de certains tableaux de genre néerlandais, conçus comme un puzzle moral dont la nature évasive continue à défier les historiens d'art de notre époque. Un résumé succinct de quelques motifs moraux ayant cours et leur utilisation par le dramaturge peut donner une idée des parallèles éclairants entre concepts visuels et le texte dramatique.

Le motif populaire du bouffon dans l'art graphique et dans la littérature, tel le bouffon observateur dans le Fils Prodigue de Karel Van Mander (ill. II) ou dans le dessin d'Holbein, a déjà été relevé brièvement. Beaucoup de bouffons shakespeariens reflètent les traits de leurs doubles graphiques ${ }^{28}$. Le bouffon en tant que commentateur ou persona satirique a été étudié de façon étendue sous l'angle des traités humanistes. Cet argument majeur chez Shakespeare lié à celui de la moriomachie ou "great stage of fools» avait un corollaire pictural important également présent dans les écrits du dramaturge, la folie de la vertu. En effet, les aléas de certains des héros mais surtout des héroïnes shakespeariennes reflètent les représentations picturales de la folie de la vertu. Les considérations graphiques peu flatteuses de Dame Monde se trouvaient contrebalancées par les images des vertus guères appréciées dans son royaume ${ }^{29}$. Ainsi, Cordelia « redeems human nature from the general curse » (King Lear, IV.v.201). Le motif iconographique des vertus malmenées ou en exil était commun au XVI ${ }^{\mathrm{e}}$ siècle ${ }^{30}$. Conséquence tragique des aspects immoraux du monde à l'envers, le concept était appliqué à un nombre considérable de vertus

\footnotetext{
28 Josée Nuyts-Giornal, «Le Miroir de la folie, La gravure néerlandaise et le drame élisabéthain », Thèse de doctorat de l'Université Paul Valéry-Montpellier III, 1998, chap. III. ${ }^{29}$ Voir par exemple Danse autour du Monde de Pieter Balten, rappelant Stultorum Chorea de Frans Hogenberg, proche des représentations de danseurs de morisque; ou Dame Monde de Crispijn de Passe, 1599.

${ }^{30}$ Op.cit. ${ }^{\circ} 28$, p. 239-242.
} 
depuis les temps classiques ${ }^{31}$. La négligence ou le mépris des vertus et le péril auquel, par conséquent, elles sont soumises est un topos shakespearien important. Le bouffon du roi Lear utilise l'image de la Vérité malmenée (I.iv.109). Dans Pericles, le dramaturge semble se moquer de la propension contemporaine pour l'aphorisme moral ou «pretty moral». Le quiproquo crée entre Périclès et les pêcheurs à propos d'une autre vertu, l'Honnêteté, dont, dans ce bas monde, on préfère ne laisser le soin à personne, repose sur une certaine familiarité avec la sagesse proverbiale et picturale du moment ${ }^{32}$. Dans ses pièces, Shakespeare décrit certains de ses protagonistes comme étant trop nobles pour ce monde. Ces «moral» ou «poor fools», comme dans King Lear, persistent à vouloir écouter leur conscience envers et contre le courant des valeurs mondaines. Aussi, sans toujours épouser exactement les contours d'une vertu personnifiée, leur situation proverbialement délicate est systématiquement évoquée ${ }^{33}$. Celle-ci fait parfois partie de l'intrigue choisie. En effet, dans Othello, Iago se sert de la bonté de Desdémone afin d'obtenir la perte du général. «So will I turn her virtue into pitch, And out of her own goodness make the net that shall enmesh them all » (II.iii.351). Le lieu commun moral est cité de façon exhaustive dans Macbeth. Ainsi, Lady Macduff est amenée à tirer la conclusion suivante :

LADYMACD. I remember now. I am in this earthly world, where to do harm is often laudable, to do good sometime accounted dangerous folly.

(IV.ii.74)

La leçon de morale à l'intention de son fils, traitant des menteurs et de gens honnêtes précédant cette réplique, avait reçu comme réponse une

\footnotetext{
${ }^{31}$ Dans Concord, Peace and Love, une gravure sur bois de Cornélis Anthonisz, la Concorde est représentée à l'écart d'une ville en ruine. L'Amour et la Paix s'enquièrent après son malaise. Les inscriptions expliquent que ni le clergé ni la société ne se préoccupent d'elle. Dans une autre image, l'artiste montre la Vérité, une serrure sur la bouche, menacée par un soldat et mal défendue par la Peur. Cf. Christine Megan-Armstrong, "Virtues in Exile », The Moralizing Prints of Cornélis Anthonisz, New Jersey, Princeton University Press, 1990. Ainsi dans Summer's Last Will and Testament de Thomas Nashe : "Oh where dwells faith or truth!» (500) ou dans Old Fortunatus de Thomas Dekker, où la vertu se lamente : «I am banished from the earth. [...] Virtue is exiled from every city / Virtue is a fool, Vise only is wise » (I.III). Chez Shakespeare, le protagoniste principal de Coriolanus désire être oublié comme ces vertus (II.iii.55).

32 Pericles, II.i.53. Cf. op. Cit. n ${ }^{\circ} 28$, p. 289-293.

33 Orlando dans As You Like It; Antonio dans The Merchant of Venice; Albany, Kent, Cordelia dans King Lear; Desdemona dans Othello; Hamlet, Brutus, Hector. Dans King Lear, Edmund reprend, de façon hypocrite, le lieu commun à son compte: «that I must repent to be just » (III.v.12).
} 
variante sinistre du Stultorum numerus est infinitas. En effet, le garçon fait remarquer que par rapport aux gens honnêtes les menteurs sont largement en surnombre.

Comme la devinette du Bouffon dans King Lear l'indique, le concept de la folie de la vertu divise l'humanité entre les deux figures emblématiques que sont le knave et le fool. Une gravure d'après Bruegel l'Ancien, le Misanthrope (ill. vI), faisant partie d'une série de proverbes en tondo, propose une lecture similaire. Une figure solitaire portant un long manteau tourne le dos au monde tandis qu'une figure courbée dans le globe terrestre lui vole sa bourse. Le personnage courbé était déjà représenté dans Les Proverbes ou le Monde à l'envers (1559), illustrant l'idée qu'il faut savoir se courber afin de traverser le monde où la droiture n'est pas de mise. Écho du «praise in the art of stouping » dans Summer's Last Will and Testament, l'image illustre également les observations ironiques de la part de Shakespeare tel que «false hearts should never have sound legs or supple knees ${ }^{34}$ » dénonçant le comportement fourbe des flatteurs. La silhouette mélancolique dans la gravure est en deuil de voir le monde qui en tant de fraudes abonde. Geffrey Whitney emploie une image similaire dans A Choice of Emblems (1586). Un promeneur solitaire tourne le dos au monde accompagné du texte suivant: «Adue deceiptful worlde, thy pleasures I detest». La droiture dans l'art graphique figurait un comportement vertueux et honnête, attitude que le Bouffon conseille aux Knaves d'éviter, seuls les fous encombrés d'une conscience morale ne peuvent s'empêcher d'être vertueux ${ }^{35}$. Comme Bruegel l'Ancien, Shakespeare semble suggérer que ceux souhaitant être vertueux ne pouvaient ignorer un Monde fallacieux. Ainsi, «virtue itself of vice must pardon beg » (Hamlet, III.iv.153).

Érasme paracheva son Éloge de la folie avec la folie de la foi selon saint Paul. Les représentations des vertus exilées ou malmenées rappellent les Ecce Homo de Jérôme Bosch, où le Christ est montré cerné par la multitude sardonique. Le paradoxe chrétien oppose valeurs mondaines et essence divine. La pompe et les apparences du monde temporel se révélaient n'être rien du point de vue sub specie aeternitatis. Mais cette position privilégiée accordée aux spectateurs

\footnotetext{
34 Voir Timon of Athens, Troilus and Cressida.

35 Cependant dans Everyman Out of His Humour de Ben Jonson, la droiture excessive du cynique est ridiculisée comme étant une attitude hypocrite.
} 
des Corpus Christi Plays semble perdre progressivement de son évidence morale artificielle. L'ambiguïté et l'équivoque facétieuse du discours et de l'imagerie humaniste ${ }^{36}$ traduisent le doute et l'incertitude d'un $X I^{\mathrm{e}}$ siècle en proie aux bouleversements religieux. Dans une série d'estampes, Maarten van Heemskerck dépeint le monde comme un cheval sans bride désarçonnant la Justice, puis entraînant dans sa perte l'Amour et la Sagesse coiffés d'un bonnet d'âne, qui tentent de le retenir ${ }^{37}$. L'estampe Elck ou Chacun/Everyman (1561), d'après Bruegel l'Ancien (ill. VII), montre l'aveuglante obsession matérialiste de l'homme comme étant la principale raison de la course folle du Monde ${ }^{38}$. Ce que recherche fébrilement Chacun encombré de ballots parmi des tas de marchandises, c'est le privatum commodum. Les lunettes et la lanterne que porte Chacun dénotent sa folie. Shakespeare emploie le même propos dans King John, lorsque Philip the Bastard donne une description très précise de "commodity - the bias of a mad world » (II.i.562-599), même si le protagoniste reconnaît de façon ironique que son indignation moraliste provient du fait qu'il n'a pas sa part des richesses.

Un thème pictural et littéraire lié à la précédente se retrouve à maintes reprises chez le dramaturge. Il s'agit du cycle des vicissitudes humaines. Maarten van Heemskerck a illustré le concept dans une série de gravures somptueuses (ill. IV) ${ }^{39}$. Ses dessins étaient destinés à servir de modèle pour une procession organisée par la chambre des rhétoriciens d'Anvers en 1561. La série propose une somme complexe des agissements humains traversant sept phases différentes. De la Prospérité procède l'Orgueil, de l'Orgueil procède l'Envie, de L'Envie procède la Guerre, de la Guerre procède la Pauvreté, de la Pauvreté

\footnotetext{
36 Josée Nuyts-Giornal, «Savoir moral et littérature dramatique», Théâtres du Monde, $\mathrm{n}^{\circ} 11$, Avignon, Université d'Avignon, 2001, p. 27-39.

${ }^{37}$ Ilja M. Veldman, De Wereld Tussen Goed en Kwaad, Late prenten van Coornhert, La Haye, SDU, 1990, p. 58-62. Il est vrai que la Justice s'y prend mal, car elle était assise à l'envers sur sa monture, puis ne tenait ni mors ni rêne, mais ses peines semblent vains de toute manière car dans la série appelée Le monde ne se laisse brider, $1^{\mathrm{e}}$ éd. 1550, le Monde est décrit comme sot. Thomas Dekker illustre le thème de la folie de la vertu dans Old Fortunatus. Dans cette pièce, la vertu et ses acolytes entrent en scène coiffés d'un bonnet d'âne.

${ }^{38}$ Dans L'Éloge de la Folie, Érasme désigne Plutus comme le père de la folie mondaine dirigeant affaires publiques et privées. $O p$. cit., $\mathrm{n}^{\circ} 20$.

${ }^{39}$ The Triumph of the World, la première gravure de la série The Cycle of the Vicissitudes of Human Affairs (1564), de Cornélis Cort d'après Maarten van Heemskerck, a été citée par Anthony Wells-Cole comme source pour une cheminée sculptée à Chipchase Castle, Northumberland, vers 1630. Op. cit., n ${ }^{\circ}$ 6, p. 192-193.
} 
procède l'Humilité, de l'Humilité procède la Paix et de la Paix procède la Prospérité. Les personnifications sont dépeintes presque littéralement comme engendrant leurs successeurs. Également appelé le Cycle de la Guerre et de la Paix, la série de Heemskerck illustre l'image exprimée dans Timon of Athens: "Make war breed peace, make peace stint war » (v.iv.80). La première gravure d'une série sur le thème d'après Jacques de Gheyn, dévoile la relation que le concept entretient avec le motif traditionnel de la roue de la Fortune (ill. v). Cornélis Anthonisz illustra le propos dans une suite de douze personnifications, appelé The Misuse of Prosperity. Argument fort apprécié, Hans Holbein l'adapta dans deux tableaux allégoriques, Le Triomphe de la Pauvreté et Le Triomphe de la Prospérité, destinées au Merchants Hall à Londres, desquels subsistent un dessin et quelques copies. À travers le vœu pieu des marchands, exprimant le désir de retenir la Prospérité en équilibre, apparaît la dénégation ironique de l'artiste.

Il semblerait que les gravures détaillées consacrées au thème permettent de mieux appréhender ses récurrences dans le texte dramatique, que les traités et textes connus sur le sujet ${ }^{40}$. Ainsi, Shakespeare donne une caricature indubitable du concept dans Coriolanus. La Paix y est décrite comme une léthargie engendrant rimailleurs, enfants bâtards et cocus. Ce qui constitue un résumé ironique mais à peu près exact des dessins de Maarten van Heemskerk. Le passage se parachève sur un épitomé du thème, "The wars for my money » (IV.v.218). Une description plus orthodoxe est proposée dans Pericles, concluant avec l'admonition de ne pas abuser de "plenty's cup » (II.iv.21-55). Mais le concept figuré par les estampes permet également d'éclairer certains passages ambigus comme «Welcome to the sour cup of prosperity. / Affliction may one day smile again, / till then sit thee down sorrow» (Love's Labours Lost, I.i.287) ou plus simplement les «fat ribs of peace» (King John, III.iii.8). Des allusions isolées au motif connu sont récurrentes ${ }^{41}$. Dans Hamlet « in the fatness of these pursy times» (III.iv.152) et son écho plus loin «This is the imposthume of much wealth and peace », concourent à instaurer l'impression de décrépitude morale environnante habitant le

\footnotetext{
${ }^{40}$ Op. cit., $\mathrm{n}^{\circ} 28$, p. 257-285 et 354-368.

${ }^{41}$ Références dans Pericles, les deux parties d'Henry IV, Troilus and Cressida, King John, Richard III, parmi d'autres.
} 
protagoniste. Shakespeare pousse jusqu'à l'exacerbation la pensée et les conventions morales humanistes dans son portrait du Prince mélancolique. La plupart des textes shakespeariens portent la trace d'une vision du monde empreinte de scepticisme humaniste, monde à l'envers présent dans la gravure morale, exposant ses côtés tantôt burlesques tantôt tragiques. Cependant, malgré la myriade de miroirs lui renvoyant sa folie et sa fragilité, la question renaissante de la responsabilité de l'homme reste posée. Images et thèmes mentionnés jusqu'ici aideront à aborder cette pièce sous l'angle de la gravure morale.

Dans l'imagerie humaniste, le bouffon est sage car il confesse sa propre folie. Le fait d'être «deprived of ordinary sense and reason », comme le poète dans la satire Mother Hubbards Tale, lui donne la licence de pointer et réprimander la folie cachée chez d'autres. Dans l'estampe de Hondius d'après Bruegel l'Ancien (ill. III), deux bouffons s'amusent tandis qu'un troisième se tient prêt à utiliser une poignée de cravaches, illustrant l'idée que la folie se châtie elle-même. David Vinckboons dessina un autre bouffon cruel frappant les spectateurs d'une scène de théâtre avec une marotte ou vessie dans un tableau de kermesse, rappelant littéralement Shakespeare dans As You Like It :

He that a fool doth very wisely hit Doth very foolishly, although he smart,

Seem aught but senseless of the bob. If not,

The wise man's folly is anatomiz'd

(II.vii.50-6)

Hamlet utilise ce propos particulier afin de justifier la pièce dans la pièce, The Mousetrap, aux yeux de Claudius ${ }^{42}$. Cependant, si le miroir de la folie tend initialement vers une réforme sociale, le lieu commun de la folie universelle mène in fine vers une dislocation de tout savoir humain, le discours satirique humaniste y compris ${ }^{43}$. En effet, la satire morale contient une faille conséquente, la vision humaine inepte de

\footnotetext{
42 «HAMLET. The play is the thing / Wherein I'll catch the conscience of the King» (II.ii.607).

43 Elle débouche sur l'interrogation pascalienne, également posée en termes de perspective : «Et, il n'y a qu'un point indivisible qui soit le véritable lieu, les autres sont trop près, trop loin ou trop bas. La perspective l'assigne dans l'art de la peinture. Mais dans la vérité et la morale qui l'assignera ? Blaise Pascal, Pensées, éd. Michel Le Guern, coll. Folio Classique, Paris, Gallim ard, 1977, p. 71.
} 
celui qui la pratique ${ }^{44}$. Dans l'art pictural comme dans l'art littéraire, des troubles de la vision, scornful ou warped perspective, squint-eyed soul liée aux valeurs mondaines étaient habituellement invoqués afin d'expliquer les conclusions moralement erronées des protagonistes ${ }^{45}$. De là, les lunettes de Chacun dans l'estampe attribuée à Bruegel (ill. VII). Ce dernier a su répondre de façon ingénieuse à la question profondément aliénante sur la faillibilité de la perception humaine. Dans un petit tableau à l'arrière-plan de Elck, l'artiste a dessiné un bouffon, nommé Niemat, Nemo, ou Nobody se contemplant dans un miroir portant l'inscription : « Nobody knows himself. » Aucun homme n'est conscient de ses imperfections face à la perfection de Dieu. Le miroir reflète l'idée mystique du rien, l'essence divine dont tout homme est dépositaire selon la foi chrétienne ${ }^{46}$.

Ainsi, Hamlet est destiné à devenir son propre traître, en écho au «merely our own traitors » dans All's Well That Ends Well, même si le dramaturge semble de prime abord attribuer le rôle d'observateur humaniste privilégié à son protagoniste. En effet, le héros humaniste est conscient de sa propre faillibilité, prêt à se reconnaître dans une femme peu courageuse en proie au doute, conscient d'un monde fait d'apparences fallacieuses. En tous les cas, Hamlet semble bien s'attribuer ce rôle en endossant l'habit du fou. Le Prince mélancolique va prétendre être tendre («kind») tout en étant cruel, confrontant ses victimes à leur folie pour qu'elles puissent s'amender à la manière des bouffons cruels de l'art pictural. Mais Hamlet oublie que la folie que vient dénoncer le fou est d'abord la sienne. Ainsi, Hamlet sera le meurtrier d'un père et sacrifiera «the fair forehead of an innocent love and sets a blister there », l'amour entre lui et Ophélie, traitant la vertu d'hypocrite (III.iv.41-44). Le méprisant «thou wretched, rash intruding fool» à l'adresse de Polonius poignardé peut légitimement lui être

\footnotetext{
44 Satire IIII de John Donne constitue en partie une interrogation de l'assise morale de celui qui comme le bouffon attitré «spits on he cares not although his face be as ill as theirs which in old hangings whip Christ »(220-30). Cette question morale semble également au cœur des certains autoportraits dubitatifs, tel que la scène de Crucifixion par Rembrandt où l'artiste s'est peint sous les traits d'un des hommes érigeant la croix.

45 All's Well That Ends Well, «scornful perspective». Thomas Dekker emploie les termes «squint eyed age» ou «squint eyed soul». Ainsi, John Donne, Sermons, «to look after God and Mamon is a squint eye.» Rappelant l'anamorphose dans les Ambassadeurs d'Holbein, des lunettes figurent cette vision uniquement temporelle erronée dans la gravure morale.

46 Josée Nuyts-Giornal, «King Lear's Reflection in the Mirror of Nobody: An Iconographical Question », Cahiers Élisabéthains, n 54, octobre 1998, p. 55-73.
} 
retourné. Hamlet partage certains de ses traits avec Jaques dans As You Like It. Car, malgré sa réticence affichée, il estime être né pour mettre les choses à l'endroit. "Oh cursed spite / That ever I was born to set it right!», ce qui semble bien la première indication sérieuse de sa folie. Par la suite, le Prince est prisonnier de sa perception subjective des choses, incapable de faire la différence entre un pécheur pénitent et son oncle déjà condamné, puis entre sa mère et la vertueuse Ophélie. Hamlet, au premier abord lucide, est entraîné malgré lui dans les excès de sa disposition mélancolique. Celle-ci finit par l'aveugler, alors, tel Bertram, le prince souffre d'une «scornful perspective». Ainsi, Bruegel dépeint la figure solitaire du Misanthrope portant son regard sur ce bas monde et ne daignant pas lever les yeux vers le ciel (ill. VI). De manière similaire, Hamlet ignore la vertu et l'essence divine dont chaque homme est dépositaire tout comme la Providence. Dans la pièce de Shakespeare, les conceptions éthiques erronées d'Hamlet précipitent la tragédie.

L'évolution morale du protagoniste semble ensuite indiquée par l'image de son retour «dénudé » sur les rivages de Danemark. En effet, la lettre d'Hamlet est lue par Claudius « You shall know I am set naked unto your kingdom », après quoi le Roi répète le terme naked suivi de près d'un «can you devise me? ». Une allusion probable au nuditas temporalis, dénué de toute possession et attachement temporel, figuré par Nobody, condition qui mène vers la vérité, souvent représentée nue. Le thème figure également dans une série d'estampes appelée L'échelle de Jacob d'après Maarten van Heemskerck. Un homme nu conscient de ses errements se contemple dans un miroir, une poignée de verges à la main. Ainsi, Hamlet a abandonné l'habit du fou. Dès lors, la signification première de la scène du cimetière ne semble pas tant l'idée commune du memento mori, ou quintessence of dust, symbolisé par le jeune homme avec le crâne, mais l'allusion à la moriomachie. Aussi, il est cocasse qu'Hamlet ne reconnaisse pas le crâne de Yorick. N'importe quel crâne, y compris celui d'un grand homme (Alexandre, Jules César) peut donc être le crâne d'un bouffon. Dans une réplique facétieuse, le clown avait déjà conclu que la folie d'Hamlet ne se verrait pas en Angleterre car les hommes y sont aussi fous que lui (v.i.151). Lorsque Hamlet faisait semblant d'être fou, il souffrait déjà de la folie 
de tous les hommes, mais préférait l'ignorer ${ }^{47}$. Désormais, «to know a man well were to know himself », mais il y a une providence particulière même dans la chute d'un moineau ${ }^{48}$. Shakespeare semble démontrer la conscience de la folie comme un des chemins possibles vers la rédemption. Après ce portrait moral d'un prince mélancolique, le dramaturge dépeint un misanthrope, un vieillard sénile, suivi des portraits d'un tyran sanguinaire puis d'un libertin avec sa concubine afin de convaincre de cette condition commune. Cependant, dans les pièces ultérieures, le désarroi aigu d'Hamlet semble faire place à plus de compassion, et, dans l'épilogue de The Tempest, l'auteur invite chacun à faire de même :

As you from crimes would pardoned be,

Let your indulgence set me free.

(Épilogue, 19-20)

Dans le domaine de l'art pictural comme dans celui de l'art dramatique, le discours moral se trouve travesti par le plaisir du regard et par le goût pour les sous-entendus malicieux ou tragiques. La multiplicité des détails souvent déroutants continue cependant à conférer une heureuse confusion qui se prolonge dans les jeux dramatiques par de multiples diffractions du sens, raillant par avance toute tentative d'explication systématique et épousant par là même la figure polyédrique de la vérité tant recherchée.

Josée Nuyts-Giornal

\footnotetext{
47 À partir de cette scène, Hamlet se montre plus honnête envers lui-même tout comme envers son amour pour Ophélie, en acceptant son identité partagée avec le reste de l'humanité. Cette perspective plus large, dont le protagoniste est dès lors capable, par opposition en quelque sorte à l'idée du Danemark comme une prison, proche de la vision sub-specie aeternitatis, est indiquée par l'allusion aux étoiles devenues les «wonderwounded hearers » de son histoire.

48 «There is a special providence in the fall of a sparrow.» Le scepticisme humaniste tel celui exprimé par Montaigne semble trouver son écho dans le proverbial: «Who knows himself despises, the self despiser god advises.» Cf. Tilley, op. cit.
} 